\title{
Differenz
}

Revista internacional de estudios heideggerianos y sus derivas contemporáneas

AÑO 6 NÚMERO 5: JULIO DE 2019. e-ISSN: 2386-4877 - DOI: 10.12795/DIFFERENZ.2019.I05.06

[pp. 121-138]

Recibido: 03/02/2019

Aceptado: 25/06/2019

\section{Fenomenologia e fede nel pensiero del primo Heidegger}

\section{Phenomenology and Faith in Early Heidegger}

\author{
Fabio Carlisi \\ Universidad de Córdoba
}

\section{Resume:}

Perché Heidegger dopo il richiamo all'esigenza metodologica di una fondazione della filosofia intesa come scienza pre-teoretica originaria, in aperta polemica tanto con la scuola di Marburgo, quanto dallo storicismo di Dilthey, si rivolge al proto-cristianesimo? Perché proprio a Paolo di Tarso? Quale legame sussiste tra l'esperienza fondamentale della fede e il compito designato di una filosofia intesa come scienza pre-teoretica originaria? La nostra ipotesi di lettura, consiste nel sostenere come proprio la radicalizzazione fenomenologica, riceva dall'analisi dell'esperienza religiosa Paolo, una possibilità di esplicitazione, che rispetto ai traguardi metodologici raggiunti nei precedenti corsi soddisfa nel merito le esigenze tematiche dell'esperienza originaria pre-teoretica.

Parole-Chiave: Attuazione (Vollzug); Indicazione formale (Anzeige formale); Temporalità (Zeitlichkeit); Parusia; Fede. 


\section{Abstract:}

Why does Heidegger, after referring to the methodological need for a foundation of philosophy understood as original pre-theoretical science -in open controversy with both the Marburg school and Dilthey's historicism- turns to proto-Christianity? Why precisely to Paul of Tarsus? What link exists between the fundamental experience of faith and the designated task of a philosophy understood as original pre-theoretical science? Our hypothesis: Heidegger is trying a phenomenological radicalization. Interpreting Paul's analysis of religious experience as a possibility of explication which -compared to the methodological goals achieved in the previous courses- satisfies the thematic needs of the original pre-theoretical experience.

Keywords: Enactment (Vollzug); Formal Indication (Anzeige formale); Temporality (Zeitlichkeit); Parusia; Faith.

\section{La complessità della situazione ermeneutica}

Come ormai viene riconosciuto unanimemente dalla critica bibliografica, il riferimento al cristianesimo delle origini assume nella prospettiva di pensiero di Heidegger un'importanza, che per incidenza e intensità, può essere solo paragonabile, all'influenza che la filosofia aristotelica ebbe in seno all'economia di pensiero dello stesso Heidegger. Chi si accosta alla lettura fenomenologica delle lettere paoline e del X libro delle Confessioni di Agostino, tenendo conto, da un lato, delle modalità esplicative che lo stesso esercitava nei precedenti corsi del '19 e dall'altro, il tentativo compiuto di un'ermeneutica dell'effettività che si concretizza nei corsi successivi al '21, non può non costatare, la trasfigurazione tematica che la prospettiva del filosofo acquisisce, proprio grazie a questo confronto con il cristianesimo originario ${ }^{1}$.

In riferimento ai celebri corsi friburghesi sul cristianesimo delle origini del 1920/21 pubblicati nel volume 60 della Gesamtausgabe, -che per ovvi motivi circoscriveremo al solo ciclo di lezioni del semestre invernale del 1921: Introduzione alla fenomenologia della religione- non entreremo nel merito della trattazione specifica che in essa viene

1 II ciclo di lezioni dedicato all'interpretazione fenomenologica della vita religiosa condivide insieme a tutti gli altri corsi risalenti al primo periodo friburghese il destino di essere stato pubblicato postumo rispetto alla quasi totalità dell'opera heideggeriana. Il corso in questione è stato pubblicato dall'editore tedesco Vittorio Klostermann Frannkfurt am main nel 1995. Per tale motivo solo in un passato relativamente recente sono stati presi in considerazione dagli studiosi e considerati come fondamentali per la comprensione di quell'itinerario fenomenologico-ermeneutico che porta alla configurazione matura della riformulazione della Seinsfrage. 
condotta. Anzi per certi versi daremo per scontato il contenuto delle argomentazioni heideggeriane, e ci limiteremo a problematizzare una questione ben precisa: comprendere la motivazione originaria che spinge il giovane assistente di Messkirch a confrontarsi con il primo cristianesimo.

Heidegger giunge al confronto ufficiale con il proto-cristianesimo, dopo due anni di intensissima attività accademica nelle vesti di assistente di Husserl ${ }^{2}$. In questo periodo il giovane Privatdozent mostra sin da subito un suo peculiare modo di accostarsi alle problematiche filosofiche, coniando una specifica identità speculativa che si rivela sin dalla prime lezioni critica nei confronti di quelle scuole di pensiero che caratterizzano lo scenario filosofico tedesco dei primi del ‘900.

Perché Heidegger dopo il richiamo all'esigenza metodologica di una fondazione della filosofia intesa come scienza pre-teoretica originaria, in aperta polemica tanto con la scuola di Marburgo, quanto con storicismo di Dilthey, si rivolge al proto-cristianesimo? Perché proprio a Paolo di Tarso? Quale legame sussiste tra l'esperienza proto-cristiana della fede e il compito designato di una filosofia intesa come scienza pre-teoretica originaria?

Rispondere a queste domande contestualizzandole al percorso di pensiero che caratterizza l'impostazione fenomenologica da cui muove il nostro pensatore è l'obiettivo che ci proponiamo di argomentare in queste pagine. Si tratta di una questione che la critica al pensiero heideggeriano non sempre ha accertato, per così dire, direttamente a partire dalle indicazioni metodologiche che il corso in questione esibisce nella prima parte del corso ${ }^{3}$.

Se ci si accosta all'interpretazione heideggeriana dall'esterno, cioè, da un punto di vista storico-obiettivo, senza entrare nel merito di quella introduzione metodica che rappresenta a tutti gli effetti una sorta di manuale di istruzione, di precauzione metodologica per la comprensione degli esiti e della stessa direzione della ricerca, si rischia, infatti, di ridurre l'analisi di Heidegger ad una semplice critica al dogmatismo della teologia cristiana, cioè ad una critica che si rivolge esclusivamente all'interpretazione dogmatica-tradizionale del contenuto delle lettere ${ }^{4}$.

2 Per una analisi completa dei corsi di lezioni heideggeriani del primo periodo friburghese rimandiamo a Guida a Heidegger a cura di F. Volpi, Editori Laterza, Roma 2005, pp. 325-345.

3 La prima parte del corso che stiamo esaminando, Introduzione metodica. Filosofia, esperienza e fenomenologia della religiosa rappresenta, sotto molti aspetti, la sintesi della radicalizzazione fenomenologica raggiunta fino a quel momento dal pensiero heideggeriano.

4 "Il problema della dimensione religiosa nei testi heideggeriani assomiglia a una sfida o, quanto meno, a un'impresa paradossale. Basti pensare soltanto a Jean-Paul Sartre, che, da ammiratore di Heidegger, lo ha presentato addirittura- schierandolo dalla parte di Nietzsche-come uno dei pensatori 
La critica alla dogmatica cristiana, piuttosto, emerge dall'analisi heideggeriana come conseguenza, per così dire, in seconda istanza, cioè solo nella misura in cui si concretizza il tentativo di riportare l'esperienza della fede nell'ambito più esteso dell'esperienza fondamentale pre-teoretica. Solo in questa particolare accezione può essere scorto genuinamente l'atteggiamento critico nei confronti del "sistema del cattolicesimo" 5.

L'ipotesi della nostra indagine, che assume la valenza di una protasi della nostra ricerca, muove dalla considerazione che il richiamo all'esperienza religiosa dei primi cristiani è necessaria a Heidegger per esplicitare fenomenologicamente quanto egli stesso era già venuto a teorizzare nei precedenti cicli di lezione solo per differenza e opposizione rispetto all'impostazione metodologica della scuola di Marburgo e dello storicismo di Dilthey. II richiamo al cristianesimo, in altre parole, funge da paradigma fenomenologico di riferimento per tematizzare, questa volta positivamente, rispetto alle posizioni espresse nei precedenti corsi, l'istanza originaria del dimensione storico-temporale dell'esperienza e allo stesso tempo per sottolineare l'irriducibilità di questa stessa condizione alle pretese di un pensiero che procede esclusivamente sul primato del teoretico.

Dalla nostra prospettiva, quindi, l'esperienza fondamentale religiosa assume in Heidegger una funzione di esplicitazione radicale del metodo fenomenologico, in quanto libera l'impostazione della ricerca dai vincoli linguistici, metodologici e concettuali della filosofia tradizionale intesa come scienza teoretica-oggettiva. La possibilità di una esplicitazione radicale del carattere originario della vita viene esibito da Heidegger sul

atei rappresentativi della nostra epoca. Tuttavia vorrei mostrare che una simile comprensione di Heidegger come pensatore ateo può derivare solo da una appropriazione puramente estrinseca della sua filosofia". Gadamer, H.-G. I sentieri di Heidegger. Bologna, Centro editoriale dehoniano (Marinetti), 2018, p. 150.

5 Riportiamo qui di seguito alcuni passi della lettera del 9 Gennaio 1919 con la quale Heidegger comunicava alla sua guida spirituale Padre Krebs la sua motivazione e decisione di abbandonare il rapporto con il sistema istituzionale del Cattolicesimo nel quale si era formato (la lettera riportata in italiano è tratta da F. VoLPI, op. cit, p.12): "Egregio signor professore, i due anni trascorsi, nei quali mi sono preoccupato di chiarire nei principi la mia posizione filosofica tralasciando ogni compito scientifico particolare, mi hanno condotto a risultati per i quali, se stessi in un vincolo exstrafilosofico, non potrei avare garantita la libertà di convinzione e insegnamento. Intuizioni gnoseologiche, che coinvolgono la teoria del conoscere storico, hanno reso per me problematico e inaccettabile il sistema del cattolicesimo, non però il cristianesimo e la metafisica (quest'ultima tuttavia, in un senso nuovo) (...) E' difficile vivere da filosofo: I'intima franchezza di fronte a se stessi e a coloro i quali si deve insegnare esige sacrifici, rinunce e lotte che all'artigiano della scienza rimangono sempre estranee. Credo di avere l'intima vocazione alla filosofia e, attuandola nella ricerca e nell'insegnamento, credo di fare ciò che le mie forze mi permettono per la destinazione eterna dell'uomo interiore, e soltanto per essa, e così credo di giustificate da solo dinanzi a Dio la mia esistenza e il mio operato". 
terreno dell'esperienza cristiana della fede che è ad avviso di Heidegger, il terreno in assoluto più idoneo per cogliere il carattere storico-temporale dell'esperire vivente.

1.La religiosità protocristiana si dà nell'esperienza protocristiana della vita ed è essa stessa una esperienza siffatta.

2.L’esperienza effettiva della vita è storica. La religiosità cristiana vive la temporalità in quanto tale ${ }^{6}$.

L'obiettivo dal punto di vista metodologico è di seguire l'autore sul suo stesso sentiero e analizzare il rapporto tra fenomenologia e fede in modo da non rimanere intrappolati in una comprensione preconcetta del contenuto storico-obiettivo dell'interpretazione, ma di portare ad evidenza tematica l'atteggiamento conforme all'esperire attuativo che accompagna l'analisi del filosofo di Messkirch. In altri termini applicheremo rigorosamente la procedura della indicazione formale alla stessa analisi heideggeriana, per mostrare al di là del contenuto ermeneutico che l'interpretazione di per sé esibisce, un riferimento più originario, su cui si istituisce il senso della Vollzug originaria a cui la stessa indagine di Heidegger deve essere riportata per essere compresa in tutta la sua complessità. E' questo in fondo il senso di quelle precauzioni metodologiche che l'indicazione formale fornisce in merito al procedere fenomenologico?

Da questa prospettiva, quindi, appare innegabile che la direzione della interpretazione del pensatore di Messkirch può essere compresa nella sua radicalità solo se si prende consapevolezza che l'esito ermeneutico a cui lo stesso Heidegger perviene nel rapporto con l'apostolo Paolo, implica una essere coinvolto originario, per così dire, in prima persona, nell'istanza intima dell'io sono della situazione ermeneutica (Vollzugssinn) -in qualche modo prescritta nella parte metodologico-introduttiva, ma che matura solo nel confronto diretto con la situazione escatologica proto-cristiana.

La questione, ora, è se vi sia la possibilità di scoprire un altro senso di 'storico' che non possa assolutamente essere attribuito agli obietti (...) 'Storico' non indica qui semplicemente lo scorrere nel tempo cui è sottoposto un oggetto, bensì: lo storico è vitalità immediata (...) ciò che si afferma come logica storica e metodologia della storia non ha alcun contatto con la storicità vivente che si è per così dire radicata nella nostra esistenza (...) Non si dice

6 M. HEIDEGGER, Fenomenologia della vita religiosa. Milano, Adelphi, 2003. p.118. Da ora in poi M. HEIDEGGER, GA 60.

7 Cfr. Infra nota 13 e 30. 
mai: 'qualcosa è storico', qualcosa come un obietto, ha la proprietà di essere storico. Così lo storico ricade in un contesto obiettivo ${ }^{8}$.

Volendo anticipare le nostre conclusioni, se pur precariamente rispetto lo sviluppo della nostra analisi, è necessario ribadire che se da un lato, dal punto di vista storico-obiettivo il riferimento al proto-cristianesimo sembra giustificarsi in vista di una deformalizzazione dell'interpretazione teologica tradizionale delle lettere di Paolo, con l'intento di mostrare al di là del contenuto (Gehalt) l'attuazione originaria (Vollzug) che l'esperienza fondamentale religiosa dischiude. Dall'altro, la direzione di pensiero del filosofo di Messkirch esibisce, allo stesso tempo, una più radicale prospettiva ermeneutica che riguarda la stessa possibilità di accesso alla situazione ermeneutica ${ }^{9}$ in virtù della quale ogni interprete è chiamato ad assumere su se stesso e che risulta impossibile tradurre in via preliminare in termini descrittivi, cioè nella distanza del pensiero rispetto al carattere simultaneo-immanente dell'effettività della vita. Questa concreta possibilità di esplicitazione, a nostro avviso, può essere guadagnata solo impegnandoci a rimanere nell'orizzonte di senso che si dischiude nel confronto con la situazione escatologica-apocalittica che caratterizza la predicazione di Paolo.

La condizione dell'accesso alla situazione ermeneutica è, secondo la nostra lettura, il centro semantico prioritario che l'interpretazione del proto-cristianesimo di Heidegger tenta di dipanare sul terreno stesso del messaggio cristiano della Parusia, dell'essere divenuti cristiani, in un modo per cui la dimensione della temporalità proto-cristiana è consegnata alla prospettiva heideggeriana come condizione originaria dell'avere la vita, cioè come condizione modale di un rapporto-a che risulta refrattaria a qualsiasi procedura di possesso teoretico. In questo senso, nelle pagine oggetto della nostra analisi, inizia a dischiudersi l'idea, tutta heideggeriana, che i concetti legati alla tradizione filosofica occidentale, ma altresì della stessa teologia cristiana che a quella si richiama, siano di per sé insufficienti per comprendere l'originario messaggio cristiano.

8 Ivi. p. 66-69.

9 "La parola «situazione» è intesa qui come termine fenomenologico, non è cioè impiegata per contesti obiettivi (nemmeno nel senso storico di "condizione" (Lage): per esempio, situazione -ovvero condizione- disastrosa). Dunque la parola "situazione" è per noi qualcosa di inerente al comprendere conforme all'attuazione e non designa nulla di conforme a un ordine". M. HEIDEGGER, op. cit. p. 130. 


\section{Alcune precisazioni sul senso della Vollzug}

Come già avevamo accennato, nel periodo che precede il riferimento al proto-cristianesimo, Heidegger ha già sviluppato e portato alle estreme conseguenze l'impostazione fenomenologica tradizionale. In seno a questo periodo di ricerca l'autore di Essere $e$ Tempo sviluppa la propria prospettiva filosofica come scienza pre-teoretica originaria facendo emergere come il riferimento immanente-simultaneo dell'effettività della vita risulta irriducibile rispetto alle procedure devitalizzanti della formalizzazione tradizionale. In questo contesto il carattere effettivo della vita, nella sua triplice articolazione interna, um-welt, mit-welt, selbst-welt, non viene più considerato unilateralmente, cioè a partire da una prospettiva conforme all'atteggiamento teoretico, ma problematizzata soprattutto nella condizione originaria dell'attuazione. Il termine-concetto attuazione (Vollzug), tematizzato a partire dal semestre estivo del 1920, è impiegato sin da subito da Heidegger per circoscrivere quella condizione pre-teoretica che caratterizza il darsi della vita e per la quale essa esibisce una originaria integrità, non riducibile né al senso dei contenuti di cui si riempie, né al senso di riferimento teoretico che la abita come realtà trascendente. In questo modo il concetto-termine attuazione sembra essere introdotto nell'economia metodologica heideggeriana allo scopo di portare ad evidenza tematica il senso di riferimento originario che contraddistingue l'effettività della vita nella quale l'essere umano è coinvolto originariamente.

L'attuazione originaria è la modalità d'essere propria della vita, essa non è, e non deve essere confusa con un presupposto teoretico a partire dal quale poter descrivere e concettualizzare la vita. La Vollzug indica piuttosto la condizione pre-teoretica del riferimento alla vita, cioè la possibilità di accedere alla motilità costitutiva, endogena del suo darsi nella sua forma essenziale e alla sua realtà concreta. L'attuazione è la considerazione della vita in rapporto con se stessa nella libertà del suo riferimento, ovvero lontana dalla sua stessa tendenza mondana e dal costante rifuggire nella significatività. In questo senso, l'esplicazione fenomenologica dell'attuazione originaria è ciò che permette alla vita una sorta di inversione rispetto la tendenza alla decadenza presso il mondo-ambiente. Detto ancora in altri termini, l'esplicitazione fenomenologica dell'attuazione è ciò che consente di portare ad evidenza tematica quella motilità originaria a partire dalla quale la vita si riappropria di se stessa per comprenderla nella prossimità della sua scaturigine.

Il modo in cui la vita ritorna su se stessa nella vicinanza della sua origine è concepito da Heidegger non come un percorso estrinseco alla vita, ma come la possibilità più propria della vita stessa. In questo modo, il filosofo di Messkirch stabilisce tra vita e filosofia una relazione di identità-reciprocità, tale da poter considerare quest'ultima, la filosofia, come manifestazione stessa della vita che sa di se stessa e vuole se stessa, un ritorno della vita 
alla sua scaturigine, e in questo senso, filosofia come conversione (Umwandlung) della vita alla vita stessa.

In virtù di questo senso ultimo della fenomenologia della vita, che assume sin da subito sfumature ed echi soteriologici, Heidegger si confronta con il cristianesimo delle origini.

\section{Il riferimento a Paolo di Tarso}

E' bene tuttavia ribadire che il richiamo all'esperienza religiosa sembra essere motivata dallo stesso Heidegger, soprattutto, per ovviare alla precarietà metodologica della tradizione filosofica occidentale, più volte indicata nella prospettiva dei corsi friburghesi. Il ricorso all'analisi dell'esperienza di vita dei primi cristiani assume quindi un significato di rottura rispetto ai paradigmi concettuali, linguistici e metodologici della tradizione filosofica ${ }^{10}$. Heidegger è alla ricerca di un modo originario di indicare, per il quale le categorie logico-concettuali del pensiero filosofico, rappresentano i primi ostacoli da superare, affinché lo sguardo possa accedere nella direzione di tale origine e lasciarsi guidare da esso ${ }^{11}$. Nell'iter dell'analisi fenomenologica sulla vita religiosa, l'autore tedesco mostra infatti, una attenzione sempre maggiore per le modalità esplicative del vissuto vivente del predicatore -in particolare per la dimensione escatologica-apocalittica- fino al punto da trascendere il riferimento al contenuto storico tradizionale delle lettere. II rapporto con Paolo, sarebbe quindi inquadrato nella direzione di una comprensione

10 "Heidegger insiste anzitutto sul carattere anti-assicurante della fede cristiana, sul suo carattere di XXXfrattura profonda. L'entrare nella fede rappresenta l'abbandono di ogni prospettiva di assicurazione teoretica e di sicurezza razionale. Essa scompagina l'ordine del teoretico nel senso per cui rompe l'ordine su cui il primato della coscienza teoretica si fonda: la linearità del tempo". A. Le Moli. Heidegger: Soggettività e differenza. Questione dell'uomo e impegno ontologico, MilanoUdine, Mimesis Edizioni, 2011. p. 56.

11 "Impegnarsi filosoficamente con Paolo su ciò che è "storico" vuol dire presupporre non solo positivamente, che Paolo ha fatto un'autentica esperienza del tempo e che ha posto il massimo impegno nel testimoniarla e nel comunicarla autenticamente ai cristiani da lui convertiti, ma anche negativamente che questa sua esperienza di vita e di comunicazione non ha lasciato traccia nella cristianità immediatamente a lui successiva. Già nei vangeli sinottici Heidegger coglie la presenza di una concettualità non conforme anzi contraria alla novità dell'esperienza del tempo testimoniata nelle lettere di Paolo. Egli si propone appunto di portare alla luce non solo questa esperienza, ma la possibilità di trarre dall'esperienza dell'essere-divenuto (Gewordensein) cristiano di Paolo e dei destinatari delle sue lettere una concettualità idonea ad afferrare la vita nel suo divenire vivente e vissuto: e Heidegger sostiene che solo la "temporalità cristiana" è il tempo giusto, il Kairos, per un divenire autenticamente vivente e vissuto". U. REGINA, "Dal tempo cristiano alla filosofia della religione" in MolinARo, A. Heidegger e San Paolo (interpretazioni fenomenologiche dell'epistolario paolino). Città del Vaticano, Urbaniana University, 2008, p. 59. 
radicale dell'effettività della vita per la quale le caratteristiche stesse della predicazione, ma altresì le condizioni storiche dell'apostolato di Paolo rappresentano il vero centro di interesse dell'analisi fenomenologica ${ }^{12}$.

In altri termini Heidegger sembra appellarsi a quella tonalità emotiva della situazione della predicazione di Paolo in virtù della quale il come dell'esplicitazione fenomenologica della sua analisi sembra quasi infrangersi sulle condizioni effettive dell'esperire paolino, fino al punto da far sparire la distanza tra i due ${ }^{13}$. Proprio questa commistione tra l'interprete e l'interpretato sintomo di un atteggiamento (Anzeige formale) che muove nella conformità dell'attuazione originaria, ovvero in direzione di una comprensione per la quale ciò che viene compreso non può mai essere ridotto ad un contenuto oggettivo che gode di realtà in sé, ma inserisce sempre alla trasformazione dell'identità del vivente storico-effettivo, ci suggerisce che Heidegger trovi nel confronto con Paolo, nella sua inquietudine e angustia effettiva, il testimone di questa condizione originaria dell'esperire attuativo $^{14}$. $E^{\prime}$ possibile dunque sostenere, dalla nostra prospettiva, che Heidegger rivede nella condizione storica del predicatore di Tarso, il germe delle condizioni del suo stesso interrogare e la sua stessa difficoltà di intesa con la tradizione, al punto che è possibile esibire tra i due un parallelismo tematico a partire dalla vicinanza della situazione.

Paolo si trova nella spasmodica condizione di tradurre, in termini altri rispetto la tradizione ebraica, la radicalità dell'esperienza fondamentale dell'essere divenuti cristiani. Questa esperienza fondamentale, condizione dell'essere divenuti cristiani, è la fede, concepita non più come qualcosa che prescrive nella fissità esterna ed impersonale (formale) della Legge, le indicazioni della condotta e delle opere a cui i fedeli devono

12 In questo senso suggeriamo che la motivazione originaria che spinge Heidegger verso le istanze della riflessione religiosa dei primi cristiani non può essere giustificata esclusivamente nella direzione di finalità storico-ermeneutiche, ma sembra essere supportata, dalla necessità di cercare nuovi paradigmi tematici, che gli permettano una sprofondamento radicale nell'effettività della vita.

13 "Nelle considerazioni che seguono non intendiamo sviluppare un'interpretazione dogmatica o teologica, e nemmeno un'analisi storica o una meditazione religiosa, bensì limitarci a fornire una propedeutica al comprendere (Versthen) fenomenologico. II carattere peculiare del comprendere nel senso della fenomenologia della religione consiste nell'acquisire la precomprensione (Vorverständnis) per una via di accesso originaria". M. HEIDEGGER, GA 60. op. cit p. 105.

14 "Un'attuazione è originaria se, per ciò che concerne il suo senso, richiede sempre, come attuazione di un rapporto che è perlomeno genuinamente diretto anche nella modalità dell'ipseità mondana, un rinnovamento sempre attuale dell'ipseità mondana dell'esserci e, più precisamente, in modo che tale rinnovamento e la "necessità" (esigenza) del rinnovamento che in esso risiede contribuisca alla costituzione dell'ipseità mondana dell'esistenza". M. HEIDEGGER, Fenomenologia dell'intuizione e dell'espressione. Teoria della formazione del concetto filosofico (GA 59). Macerata, Quodlibet, 2012, p. 66. 
attenersi. La fede piuttosto è esperita da Paolo di Tarso, ed è su questo versante che va compreso l'interesse di Heidegger per il predicatore, come l'esperienza fondamentale che pone al centro, innanzitutto, un rinnovamento-trasformazione dell'identità della vita (conversione), per la quale, essa, la vita, non è altro che questo stesso aversi nella necessità di rinnovamento e rigenerazione ${ }^{15}$.

L'intera questione non è per Paolo una questione di conoscenza (...). Egli non dice: "In tale giorno il Signore ritornerà", e nemmeno: "Non so quando ritornerà», bensì "Voi sapete in tutta certezza...". Deve trattarsi di un sapere peculiare, poiché Paolo rinvia i Tessalonicesi a se stessi e al sapere che hanno in quanto divenuti. Da questo genere di risposta emerge che la decisione in merito alla «questione» dipende dalla loro propria vita ${ }^{16}$.

La fede non è un rapimento mistico che isola l'individuo dal mondo circostante, ma viene descritta da Paolo come un sapere (aver visto), che pur non essendo di natura teoretica, è capace di raggiungere nessi esplicativi e di essere condiviso con la collettività ${ }^{17}$. L'esperienza della fede, in altri termini, permette un'autocomprensione della vita ed una esplicazione della stessa a partire dalla sua dimensione concreta, senza categorizzarla arbitrariamente. La custodia di tale esperire essenziale nel sapere, è il motivo per cui

15 "In primo luogo diventare cristiani implica una "frattura completa con il precedente passato, ossia con ogni concezione non-cristiana della vita». Diventare cristiani significa rinunciare a ciò che si è stati, rompere la dipendenza del proprio tempo presente con la propria vita passata, spezzare la connessione significativa che collega, all'interno di ogni coscienza, presente e passato. Nella dimensione cristiana il passato assume un senso nuovo. Esso coincide con l'avvento che deve tornare, diventando quindi esso stesso futuro. II cristiano vive il tempo in una maniera paradossale, incompatibile con ogni concezione lineare o conforme ad un ordine oggettivo. Diventare cristiani significa infatti cominciare a contare il tempo a partire dalla sua fine: "la fine del tempo è già iniziata". [...] Per i Tessalonicesi, infatti: "I'essere-divenuti non è un avvenimento qualsiasi nella vita, bensì è costantemente coesperito, in modo che il loro essere attuale è il loro essere-divenuti". Ciò vuol dire che il passato forma l'attualità del loro presente in maniera diversa rispetto alla connessione causa-effetto che determina l'ordine interno alla coscienza teoretica. Heidegger afferma che "proprio riguardo a questo problema è possibile mostrare il fallimento della psicologia scientifica dell'esperienza vissuta"”. A. LE MOLI, op. cit. pp. 56-57.

16 M. HEIDEGGER, GA 60. op.cit. p. 143.

$17 \mathrm{E}^{\prime}$ importante sottolineare come la possibilità dell'essere divenuti cristiani che identifica la comunità proto-cristiana sembra essere acquisita sulla base di una situazione di appartenenza che non si concretizza su istanze teoretiche-oggettive, cioè a partire da conoscenze certe di natura cosmologica o di altro tipo. La comunità cristiana si identifica piuttosto su un avere la propria vita che richiama in prima istanza un coinvolgimento in prima persona del cristiano alla responsabilità verso se stesso libero da ogni determinazione mondana della propria esistenza. 
Heidegger intrattiene con Paolo di Tarso un confronto serrato che lo coinvolge nell'analisi appassionata delle lettere prese in esame.

Il pensatore di Messkirch, si trova nel corrispettivo filosofico della stessa condizione problematica del predicatore di Tarso, all'interno della quale, l'esperienza fondamentale pre-teoretica se non adeguatamente tematizzata nella direzione del senso dello storico -ambito per il quale l'impostazione teoretica e il linguaggio oggettivante della filosofia tradizionale non risultano essere idonei- rischia di scadere in una ipostatizzazione di una particolare regione reale, e quindi in definitiva, determinata dalla tendenza alla significatività del mondo-ambiente. Questa è la zona pericolosa della dimensione effettiva della vita ${ }^{18}$ per la quale Heidegger si accosta all'esperienza della fede descritta nelle lettere paoline, proprio al fine di esplicitare la corretta comprensione fenomenologica del senso dell'esperienza originaria nel suo intimo rapporto con le implicazioni pratiche e poietiche che questa comporta ${ }^{19}$.

Il giovane filosofo di Messkirch nonostante avesse precisato nei corsi precedenti le motivazioni fenomenologiche dell'ambito pre-teoretico dell'attuazione, nel confronto con l'apostolo, si imbatte in qualcosa di radicale che la stessa esplicazione fenomenologica dell'esperienza fondamentale pre-teoretica precedentemente elaborata, non riesce a supportare, se non rimanendo nel costante riferimento a ciò che viene dischiuso nel concetto deformalizzato della fede. Risulta paradigmatico, in questo senso, il fatto che Heidegger nel portare avanti l'analisi fenomenologica sui contenuti delle lettere, a un certo punto ritorni sulle sue stesse considerazioni metodologiche che erano state concluse nella prima parte, per una riconsiderazione più attenta dello sviluppo metodologico del Wie nella sua interdipendenza con il senso del contenuto, e il senso di riferimento ${ }^{20}$.

18 "Il concetto di effettivo non va interpretato in base a presupposti gnoseologici definitivi. Esso diventa comprensibile solo a partire dal concetto di storico. Al tempo stesso però l'esperienza effettiva della vita è una zona pericolosa per la filosofia autonoma, poiché già in tale zona si fanno valere le ambizioni della scienza". Ivi. p. 41.

19 Interessantissimo sembra il parallelismo che si può avanzare tra il senso della pericolosità insita nell'effettività della vita esplicitata da Heidegger, in cui il senso dello storico acquisito proprio dall' atteggiamento conforme all'attuazione, rischia in ultima istanza di essere autonomizzato in paradigma concettuale, e il significato che assume l'annuncio dell'anticristo come ultima prova, la prova estrema in cui i cristiani sono chiamati a decidersi.

20 "A dimostrazione della rilevanza positiva e teorica delle analisi storico-ermeneutiche di Heidegger, le considerazioni metodologiche con cui si concludeva la prima parte vengono riprese nel corso del concreto lavoro di interpretazione dei testi paolini. L'interpretazione deve passare da una connessione storico oggettiva e prefenomenologica a una situazione storica originaria, considerata nella sua concreta attuazione. Il che non significa che la messa in evidenza del "Wie", cui mira l'analisi fenomenologica, debba tralasciare i contenuti della situazione o i riferimenti in essa 


\section{L'esperienza fondamentale della fede e la fenomenologia}

Da questo presupposto diviene visibile che il motivo che spinge il filosofo di Messkirch all'analisi delle esperienze di vita dei primi cristiani, non è solo di carattere religioso, cioè non è legato esclusivamente a deformalizzare l'esperienza originaria della fede, al fine di svelare l'occultamento che questa ha subito all'interno dell'orizzonte dogmatico della teologia tradizionale. La disamina heideggeriana sembra guidata piuttosto dalla necessità di esplicitare le considerazioni fenomenologiche sulla fede, che caratterizzano l'atteggiamento di San Paolo, per chiarire l'esperienza fondamentale pre-teoretica nella sua corretta giustapposizione. Si ha l'impressione che la deformalizzazione del concetto di fede, dischiuso in stretta simbiosi con l'analisi paolina, permetta a Heidegger di liberare definitivamente il proprio interrogare fenomenologico, da un certo residuo gnoseologico -retaggio dell'eredità della fenomenologia husserliana- per impostare la questione della Vollzug, da una prospettiva ancora più radicale. Liberare il proto-cristianesimo dalla tradizione teologico-dogmatica, interpretare cioè l'esperienza della fede a partire dalle direttive fenomenologiche dell'indicazione formale, permetterebbe all'assistente di Husserl, una possibilità di esplicitazione dell'ambito pre-teoretico, che è allo stesso tempo, appropriazione-attuazione vorace, delle modalità tematiche proprie del predicatore di Tarso. In altri termini, ciò che noi sosteniamo andrebbe compreso a partire dall'esigenza heideggeriana di trovare una direzione di senso, nella quale la configurazione dell'esperienza fondamentale pre-teoretica, possa essere esibita sul terreno stesso dell'effettività -e proprio a partire da essa- in modo che in questa si possa cogliere, da un lato, lo squarcio, la fenditura che porta ad una inversione verso l'atteggiamento filosofico, rispetto alla tendenza mondana della vita, e dall'altro il fatto che tale inversione non metta in discussione i contenuti e le significatività mondane, ma che anzi le giustifichi in rapporto all'attuazione originaria ${ }^{21}$.

presenti. A differenza del teoretico, fondato sull'astrazione, l'esplicazione non elimina i riferimenti vitali che costituiscono la situazione. Senso di contenuto, di riferimento e di attuazione vengono compresi dal metodo fenomenologico nella loro unità e concretezza: L'attuazione non esiste separata dagli specifici contenuti della situazione o dai legami vitali che legano l'osservatore al contesto esaminato". L. SAVARINO, Heidegger e il cristianesimo 1916-1927, Napoli, Liguori Editori, 2001, pp. 82-83.

21 Questa intensificazione e rilevanza dello stato dell'effettività sembra essere guadagnata -e sotto certi aspetti rivalutata rispetto le stesse posizioni heideggeriane espresse nei precedenti corsi- in virtù di quella caratteristica costante della predicazione di Paolo quale l'angustia, l'inquietudine a cui è sottoposta la vita cristiana. "Paolo vive in un'angustia particolare che gli è propria in quanto apostolo, ossia nell'attesa del Secondo Avvento del Signore. Questa angustia articola la specifica situazione di Paolo. Ogni attimo della sua vita si determina in base ad essa, sicché egli, nonostante la gioia che lo anima in quanto apostolo, è costantemente sottoposto a una sofferenza. (...). Per 
Questa possibilità di esplicitazione, l'Heidegger lettore di Paolo, la coglie e la esibisce proprio in quelle argomentazioni ormai divenute celebri. Le tematiche presenti nelle lettere e ora deformalizzate da Heidegger -il rapporto tra legge e fede, il sapere dell'essere divenuti dei Tessalonicesi, l'attesa della Parusia, il senso stesso dell'apostasiahanno in comune questo denominatore fenomenologico: incalzando e radicalizzando tali esperienze, Heidegger, ridefinisce quel senso della Vollzug che aveva, nei corsi precedenti, appena abbozzato in seno alla vita filosofica, dipanando definitivamente il senso storico dell'attuazione ${ }^{22}$.

$\mathrm{E}^{\prime}$ in questo contesto teorico, infatti, che il giovane Heidegger ridefinisce l'ambito dell'esperienza fondamentale pre-teoretica, la Vollzug, nella sua intima connessione con l'escatologia-apocalittica ${ }^{23}$ cristiana, maturando quel concetto di temporalità (Zeitlichkeit) come condizione originaria di accesso all'essere dei fenomeni o, il che è lo stesso, al fenomeno dell'essere ${ }^{24}$.

Paolo il fatto decisivo non è l'avere ricevuto il dono della grazia: qui egli taglia corto e non ci dice nulla in proposito. II "come" dell'estasi è ignoto e irrilevante. (...) Soltanto quando è debole, quando sopporta le necessità della sua esistenza, egli può entrare in uno stretto rapporto con Dio. L'esigenza fondamentale dell'avere-Dio (das gott-Haben) è l'opposto di ogni cattiva mistica. Decisivo diventa non lo sprofondamento mistico, lo sforzo particolare, bensì sopportare la debolezza della vita". M. HEIDEGGER. GA 60, op. cit. p.138-140. Si comprende da tale esplicitazione come per Heidegger ci sia un legame essenziale tra l'elemento escatologico-apocalittico che angustia la vita del cristiano e l'avere rapporto con Dio. In questo senso in Heidegger si precisa un senso di effettività della vita.

22 In questo senso va compresa la nostra ipotesi che esiste una trama semantica che attraversa il quid dei messaggi delle lettere di Paolo esaminati da Heidegger, per la quale i contenuti e i riferimenti esplicitati, sono solo la conseguenza di un significato più intimo del rapporto tra Heidegger e il proto-cristianesimo.

23 II fattore escatologico e apocalittico presente nel proto-cristianesimo è considerato da Heidegger un tratto distintivo e originario di autenticità della vita cristiana, la sua mancanza, nell'atteggiamento della fede è segno di una inautentica comprensione dell'originario messaggio cristiano. "Consideriamo ora il centro della vita cristiana: il problema escatologico. Già alla fine del primo secolo l'elemento escatologico presente nel cristianesimo fu occultato, e in epoca successiva tutti i concetti originariamente cristiani furono disconosciuti. Anche nella filosofia odierna i costrutti concettuali cristiani sono ancora celati dietro l'atteggiamento greco". M. HEIDEGGER GA 60, op.cit. p. 145.

24 L'attesa del ritorno del Cristo, caratterizzata da quel senso di tribolazione in cui vive il credente -per il fatto che il tempo del mondo storico è destinato alla fine- dischiude una sorta di tempo intermedio kairos, per il quale l'attesa non implica più il "quando" obiettivo-quantitativo (Kronos), ma il come vivere, ossia, vivere il tempo originariamente. II vero cristiano è colui che abbandona la pretesa dello sguardo oggettivante sul mondo e sceglie, cioè vive il tempo dell'attesa secondo l'indicazione paolina del "come se non" in cui il senso di contenuto e di riferimento del mondo vengono trasfigurati e compresi nel tempo originario dell'attuazione. In questo senso il tempo della Parusia esplicita nel suo fondamento la motilità dell'attuazione heideggeriana: "Per il cristiano 
Sembra chiaro, quindi, che il richiamo al cristianesimo delle origini è compiuto da Heidegger perché consapevole che in esso è custodita la dimensione più originaria dell'esperienza del sé, dell'io storico, inteso come ipseità originaria (Vollzug), tematizzata attraverso una prospettiva che, in quanto lontana dalle pretese oggettivanti delle filosofie -ma anche da qualsiasi fenomenologia della coscienza a cui egli si sentiva ancora legatorisulta efficacie nel rendere l'originario orizzonte di senso dell'esperienza fondamentale pre-teoretica.

Questo intimo legame sul quale si fonda la vicinanza di pensiero tra il predicatore di Tarso e il filosofo di Messkirch, assume, quindi, un significato radicale, anche rispetto al contenuto storico obiettivo dell'interpretazione heideggeriana. L'esplicitazione fenomenologica del tempo, infatti, non esibisce soltanto la condizione originaria della dimensione cristiana della vita, ma assume nella prospettiva heideggeriana anche e soprattutto un valore di indicazione rispetto al compito del pensiero filosofico, il cui fine è la custodia di tale condizione nel sapere.

Nell'attesa di Paolo il rapporto con Dio è sempre considerato in virtù del senso di attuazione alla vita, Dio non è mai oggetto di conoscenza teoretica, e la possibilità stessa della conoscenza rientra nell'esperienza effettiva della vita. Ciò significa per Heidegger che in Paolo si concretizza la decisione di assumere la problematicità della vita, di portarla ad una radicale esplicitazione, rinunciando paradossalmente al dogma della verità rivelata, per la quale si attesta il raggiungimento e il possesso del senso stesso della vita. In ciò, Heidegger scorge la radicalità del messaggio paolino nel quale far convergere il compito dell'interrogare filosofico:

La filosofia nella sua radicale problematicità che si appoggia a se stessa deve essere in linea di principio a-teistica. Ad essa, proprio sulla base della sua tendenza fondamentale, non è lecito presumere di possedere e di determinare Dio. Quanto più essa è radicale, tanto più è lontana da lui, e

vivere significa disporsi nella dimensione dell'hos me, del "come se non", vivere il come del "non", del negativo. (...) Vivere cristianamente (vivere fattualmente) significa radicalizzare la caduta nella rete dei riferimenti mondani che intrappolano l'uomo, vivere in modo diverso la necessità del radicarsi in una rete di significatività mondane. Radicarsi nella caduta a tal punto da capire che le significatività che ci vincolano possono essere negate, reinterpretate a partire da un altro contesto di riferimento. (...) È in quest'ultimo modo che si rispetta il carattere processuale della vita e si vive la caduta necessaria nel mondo e nei suoi significati come qualcosa che non chiude ma che, aprendo sempre nuovi spazi di possibilità, permette alla vita di conservare la sua natura di movimento". A. LE MOLI. op.cit. pp. 59-60. 
per tanto proprio nel radicale compimento del "Iontano", un peculiare ed arduo essere presso di lui ${ }^{25}$.

La Vollzug -compresa ora nell'apertura temporale dell'attimo- non è qualcosa che si possiede, come si può possedere una conoscenza oggettuale-teoretica, ma è piuttosto qualcosa che si ha ${ }^{26}$. L'avere questa condizione è qualcosa che non può essere compresa né tantomeno custodita -rimanendo nell'orizzonte teoretico della conoscenza ${ }^{27}$, né d'altro

25 M. HEIDEGGER, Interpretazioni fenomenologiche di Aristotele (Introduzione alla ricerca fenomenologica), Napoli, Guida, 2001, p. 226. In questo senso va compresa a nostro avviso l'affermazione del suo considerarsi un "teologo cristiano» testimoniata al suo allievo ed amico Karl Löwith. Questa testimonianza che in tutta prima sembra entrare in conflitto con le dichiarazioni espresse a Engelbert Krebs, (Cfr. Supra nota 5) in realtà assume una convalida di ciò che Heidegger afferma il 9 gennaio 1919 in merito al non essere impegnato in condizionamenti extrafilosofici per la ricerca. Lo stesso Fabris sottolinea come l'espressione testimoniata nella lettera a Löwith vada intesa nella direzione delle assunzioni metodologiche annunciate a padre Krebs nel 1919: "In realtà tale espressione, lungi dall'indicare una scelta precisa di campo, va ricondotta invece al contesto più generale della lettera a Krebs citata, e dev'essere compresa appunto nel suo pieno significato. Essa infatti non esprime l'accoglimento da parte di Heidegger dei contenuti della religione cristiana, bensì manifesta la sua intenzione di affrontare, seguendo la via del pensiero filosofico, il logos del cristianesimo e di identificare in tal modo le strutture portanti che si riflettono nella vita religiosa. Più in particolare, la dichiarazione del proprio essere un "teologo cristiano" è volta a specificare il modo in cui Heidegger stesso vive e sperimenta la propria situazione filosofica, il modo in cui, cioè, egli è in grado di riempire di contenuti quell'"essere" coniugato alla prima persona, quell' "io sono" che enuncia nella lettera poco tempo dopo aver tenuto i due corsi di lezione su argomenti di fenomenologia della religione". A. FABRIS, L'«ermeneutica della fatticità» nei corsi friburghesi dal 1919 al 1923 in VolPI, F. Guida a Heidegger op. cit., p. 70.

26 "Come si risponde a questo tempo paradossale? Come si prende in custodia qualcosa che non è? E cosa si "ha" quando si "hanno" fede e speranza? Heidegger risponde che non si ha un "che" ma un "come". Non si hanno oggetti bensì modi. E sono questi modi a determinare ciò che si ha, proprio in quanto a partire dalla loro centralità si dissolve ogni rappresentazione teoretica del tempo e della vita. Come la vita scompare in quanto insieme di processualità causa-effettuali, così scompare il tempo in quanto realtà obiettivabile. II 'quando' del secondo avvento non è infatti un punto che stia obiettivamente alla fine del corso lineare della storia bensì un modo di rapportarsi ad ogni istante del proprio tempo. È la celebre immagine paolina del giorno del Signore che "giunge come un ladro nella notte" e rispetto al quale non ci si può disporre alcuna sequenza di operazioni in previsione di un suo accadere obiettivo ma occorre sempre essere saldi, desti e sobri perché ogni istante potrebbe essere quello della Sua venuta". A. LE MOLI, op.cit. pp.58-59.

27 "L'interpretazione heideggeriana del testo paolino raggiunge così il suo apice: descrivendo un movimento analogo a quella Umwendung in cui consiste la possibilità della vita filosofica, Heidegger rilegge in chiave filosofico-ontologica un motivo etico-cristiano. Nella misura in cui si colloca a livello dell'attuazione e non intacca i contenuti di esistenza, l'essere-divenuti attraverso la parola di Dio significa aver ricevuto la possibilità di una svolta radicale verso il se stesso autentico e verso la fatticità della vita, che va contro la tendenza rovinante-deiettiva della fuga nel mondo, di cui il teoretico è la possibilità per eccellenza". L. SAVARINO. op. cit. p. 91. 
canto Heidegger si accontenta di una possibile problematizzazione di questo piano da un versante teologico ${ }^{28}$. Ma sulle indicazioni di questo avare-da-essere, dischiuso dal cristianesimo delle origini -ma problematizzato soprattutto nel confronto con Agostino- il pensatore di Messkirch intraprende il sentiero filosofico, destinato ad essere interrotto, di una radicale fondazione ontologica di tale condizione ${ }^{29}$.

\section{Conclusioni}

Queste nostre considerazioni sull'interpretazione heideggeriana delle lettere paoline hanno voluto soltanto indicare il compito di pensiero imposto all'interprete dalla stessa analisi heideggeriana affinché si compia il senso stesso dello storico, vero traguardo del riferimento al predicatore di Tarso. Abbandonando la pretesa dei riferimenti testuali storicoobiettivi e incalzato, piuttosto, dal senso originario dell'effettivo dipanato dall'itinerario metodologico dell'indicazione formale, Heidegger si imbatte in quella situazione effettiva angustiante e tribolante che l'esperire attuativo incarna come sintomo autentico dello stare al mondo. Questa condizione originaria (Vollzug) com'è noto non può essere ipostatizzata come realtà in sé autonoma estranea al divenire vivente, ma deve essere ogni volta guadagnata attraverso l'esercizio fenomenologico della deformalizzazione (Anzeige formale) dalla quale, e sola da questa, secondo Heidegger, prende avvio una legittima esplicazione tematica dell'interpretazione come della descrizione dell'atto conoscitivo in generale ${ }^{30}$.

28 II corso del 1927 Fenomenologia e teologia sembra essere incentrato proprio sulla possibilità di istituire una filosofia del cristianesimo il cui esito tuttavia sarà negativo. Se da un lato Heidegger riconosce alla teologia, ripensata attraverso il guadagno tematico della fede dischiusa nelle interpretazione delle lettere di Paolo, una sua autonomia come scienza, dall'altro, considera dominio esclusivo dell'interrogare filosofico la liberà e il confronto con le problematiche ontologiche.

29 “Una volta guadagnato l'accesso all'effettività attraverso il cristianesimo, Heidegger si rivolge ad Aristotele, ed introduce una radicalizzazione ontologica, in base al quale l'effettivo viene a coincidere con l'ontico e con l'esistentivo, o con l'essere-gettato (Geworfenheit) e con la situazione emotiva, mentre si assiste all'assunzione crescente (sino alla maggiore evidenza in ambito temporale) dell'autenticità come carattere ontologico e originario dell'esistenziale". P. DE VITIS, "Principali interpretazioni della vorlesung heideggeriana", in A. MolinARO. Heidegger e San Paolo (interpretazioni fenomenologiche dell'epistolario paolino) op. cit. p. 112.

30 "L'indicazione formale ha un significato indispensabile all'interno della filosofia, e tuttavia esso può diventare comprensibile solo nel momento in cui l'indicazione formale e ciò che viene indicato con essa non viene ipostatizzato fino ad essere trasformato nel fine e nell'oggetto del lavoro filosofico, ma si trovi in una modalità ben definita al servizio del compito della filosofia: richiamare l'attenzione a partire dalla comprensione originaria. L'indicazione formale, cioè, è contemporaneamente motivata autenticamente da ciò che è concreto ed effettivo. Non nel senso 
In questo senso la nostra lettura delle pagine heideggeriane ha preteso, al fine di non tradire il Nostro autore proprio sul senso fondamentale della sua interpretazione, che il contenuto e il riferimento attuativo delle sue stesse riflessioni non fossero ridotte ad un semplice punto di vista accanto ad altri di una interpretazione storica, ma rese vivide nella misura in cui comprese a partire dalla condizione storico-effettiva dell'immedesimazione con la situazione escatologica paolina ${ }^{31}$. La sua stessa crisi religiosa, infatti, testimoniata, altresì, dal carteggio con la sua guida spirituale e con l'amico e allievo Löwith, poco sopra ricordata ${ }^{32}$, non è la conseguenza esterna di una riflessione radicale, ma il sintomo effettivo di quella condizione inquieta e tribolante che chi intraprende il sentiero filosofico dedito al domandare radicale necessariamente è chiamato ad assumere su se stesso. Credo che sia proprio questo il senso intimo che le pagine heideggeriane in questione hanno forza di istituire. L'indicazione formale heideggeriana non è un costrutto teorico-metodologico da applicare indistintamente, alternativo alla formalizzazione di tradizione fenomenologica di matrice husserliana, ma è l'esplicitazione fenomenologica del senso stesso dell'interrogare filosofico che, come abbiamo cercato di dimostrare, affonda le proprie radici sulla motilità "endogena e autoferenziale" che caratterizza l'effettività dell'interrogante. Solo da questa prospettiva si ha accesso in modo genuino a quella situazione del pensiero indicata nell'ermeneutica dell'effettività a partire dalla quale, come è noto, Heidegger intraprende

di ciò che è comune a ciò che è effettivo, ma nel senso di prendere contatto, privo di pregiudizi e in ogni caso mai portato a compimento in modo definitivo, secondo una pre-delineazione, con ciò che è effettivo". M. HEIDEGGER. GA 60. op. cit. pp. 99-100.

31 "Il problema dell'immedesimazione (Einfühlung) non fa un passo avanti finché lo si concepisce in termini gnoseologici, dato che il motivo di tale problema non è affatto gnoseologico. L'immedesimazione compare nell'esperienza effettiva della vita, è cioè un fenomeno storicooriginario che non può essere risolto senza il fenomeno della tradizione (tradition) in senso originario. (...) Con il compimento dell'esplicazione l'esplicato si trasforma apparentemente in qualcosa di autonomo, staccato dall'attuazione. Si tratta tuttavia di un'idea sbagliata. All'astrazione teoretica, conforme all'atteggiamento, è peculiare che ciò che è stato astratto sia concepito come momento di una regione reale, sicché in tal modo è acquisita la determinazione fondamentale della regione. Ciò che è stato astratto viene ulteriormente considerato senza badare a ciò da cui è stato astratto: il fundamentum dell'astrazione è indifferente. L'astrazione come tale -il passaggio dal fundamentum abstractionis all'astrarre- non giunge a essere co-esperita. Diverso è il caso dell'esplicitazione: quando nell'esplicazione sono esplicati determinati momenti, i momenti di senso verso cui l'esplicazione non si dirige non sono messi da parte, ma il "come" del loro emergere nella direzione di senso appena esplicata -ovvero concepita nell'esplicazione- risulta simultaneamente determinato proprio in virtù dell'esplicazione stessa. (...) L'attuazione dell'esplicazione non è una sequenza discontinua di atti, cioè di determinazioni del coglimento (Erfassungbestimmungen). Essa può essere ottenuta solo in un contesto concreto della vita". Ivi. pp. 124-125.

32 cfr. Supra nota 5 e 25. 
il cammino per una nuova formulazione della Seinsfrage esibita nella sua forma più matura nell'ontologia fondamentale di Essere e Tempo.

\section{Bibliografía}

P. DE VITIS, "Principali interpretazioni della vorlesung heideggeriana", in A. MOLINARO. Heidegger e San Paolo (interpretazioni fenomenologiche dell'epistolario paolino). Città del Vaticano, Urbaniana University, 2008.

GADAMER, H.-G. I sentieri di Heidegger. Tr. R. Cristin. Bologna. Centro editoriale dehoniano (Marinetti), 2018.

HEIDEgGeR, M. Phänomenologie des religiösen Lebens. GA 60. Frankfurt am Main, Klostermann, 1995 (Fenomenologia della vita religiosa. Tr. G. Gurisatti. Milano, Adelphi, 2003).

HEIDEGGER, M. Fenomenologia dell'intuizione e dell'espressione. Teoria della formazione del concetto filosofico (GA 59). Tr.. A. Canzonieri. Macerata, Quodlibet, 2012.

HEIDEGGER, M. Interpretazioni fenomenologiche di Aristotele (Introduzione alla ricerca fenomenologica). Tr. De Carolis. Napoli, Guida, 2001.

LE MOLI. A. Heidegger: Soggettività e differenza. Questione dell'uomo e impegno ontologico, Milano-Udine, Mimesis Edizioni, 2011.

REGINA, U. "Dal tempo cristiano alla filosofia della religione" in MolinARO, A. Heidegger e San Paolo (interpretazioni fenomenologiche dell'epistolario paolino). Città del Vaticano, Urbaniana University, 2008.

SAVARINO, L. Heidegger e il cristianesimo 1916-1927. Napoli, Liguori Editori, 2001.

VoLPI, F. Guida a Heidegger. Roma, Laterza, 2005. 